\section{JOIN CAREER DAY AT COLOGNE 2015}

More than 2,000 companies from about 60 countries are expected to attend the International Dental Show (IDS) in Cologne from 10-14 March 2015. Taking place every two years, the IDS is the world's most important get-together for the international dental market.

This year, IDS Career Day is the meeting point between exhibitors and new talent. Companies use the IDS to address students, graduates, trainees and those interested in a career in the dental profession in a targeted manner.

The IDS Career Day will take place on Saturday 14 March 2015 in the Speaker's Corner area of Hall 3.1. Participants from far afield can take advantage of overnight accommodation in the Cologne-Deutz youth hostel nearby on the weekend of 13/14 March 2015. Subject to availability, rooms can be requested and booked by quoting the reservation number 178137.

Exhibitors can book brief presentation slots to introduce your company to delegates in Hall 3.1 and a recruitment lounge connected to the presentation area will offer facilities for individual meetings and presentations in partitioned areas. All exhibitors involved in the Career Day initiative will be named on IDS homepage and on a special printed flyer.

To find out more visit http://english.ids-cologne.de/ids/index-2.php.

\section{RECRUIT DCP STAFF FOR FREE ONLINE}

British Dental Association (BDA) members can now advertise their dental care professional (DCP) roles for free online at bdjjobs.com, the $B D J$ 's dental jobs and classified listings website. Qualifying DCP roles include dental nurses, practice managers, dental technicians, and receptionists, but exclude dental hygienists and dental therapists.

The starting price for DCP advertisements is normally £107.50 + VAT, so this represents a great opportunity to promote your DCP vacancies to the 29,051 (WebTrends data 2014) dental professionals that, each month, visit bdjjobs.com, which is also cited as the most used online resource for dental job searching (QA Research, Dentists' ICT Use, 2011).

To make a booking or for more information contact the BDJ Jobs sales team atbdj@nature.com or on +44 (0)20 78434729.

\section{ACCURATE COMPUTERISED CHARTING}

Periodontal exams are a fundamental part of patient care and now there is a more accurate and efficient way to gather this essential data. The Florida Probe from Clark Dental provides you with a valuable communication and education tool for the patient as it calls out numbers and messages, whilst enabling you to maximise productivity in the practice.

Only one user is required to carry out a periodontal exam thanks to the revolutionary new VoiceWorks voice controlled charting system, meaning dental nurses can efficiently use their skills elsewhere whilst the data are gathered. Computerised charting with the Florida Probe, combined with a patient diagnosis sheet, also becomes a legal document of informed consent. Introducing a system that charts and records accurately will protect your staff and the practice for the future.

Contact Clark Dental on 01268733146 or visit www.clarkdental.co.uk.

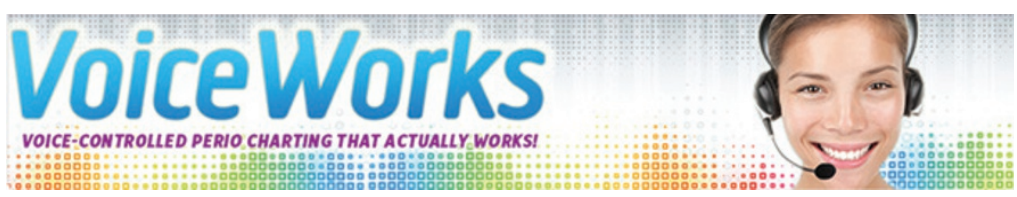

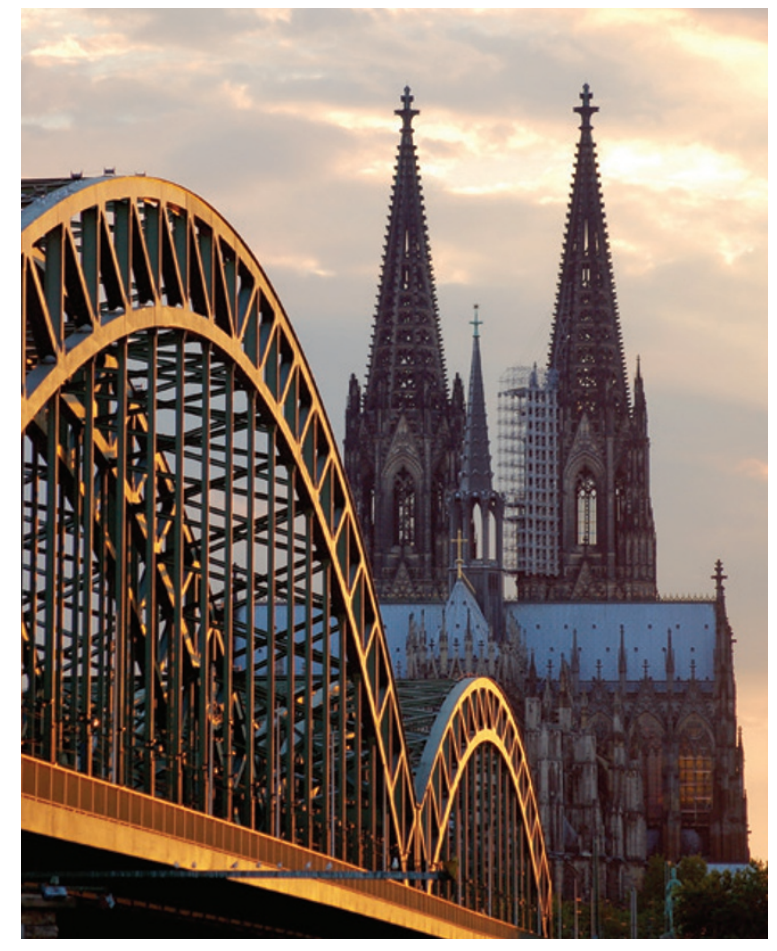

\section{HEALTHY ORAL HYGIENE IMPLANT KIT}

Failure of dental implants can not only cause patient disappointment but also lead to mistrust in their dental professional if the importance of a good oral hygiene regime is not emphasised. Tandex offers an implant kit that will enable patients to maintain a healthy oral hygiene regime before. The kit includes:

- A Tandex SoftTouch toothbrush, for delicate and sensitive cleaning in the first few days after surgery

- A Tandex Advance Soft toothbrush - for when the gingiva has healed

- A Tandex SOLO Ultrasoft - for cleaning in and around the interdental spaces

- Four FLEXI UltraSoft interdental brushes

- A Tandex Gel for reducing plaque formation, gingival inflammation and the incidence of caries.

By ensuring your patients have all the necessary equipment and guidance you will be protecting the longevity of their implants.

For more information on Tandex's products call +4547381001 or visit www.tandex.dk.

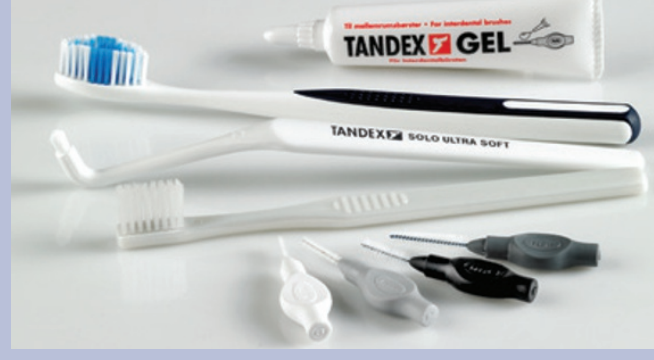

